\title{
Relation entre n-3 et n-6 avec la dépression clinique : résultats de la Nurses' Health Study
}

\author{
Michel LUCAS \\ Fariba MIRZAEI \\ Éilis J. O'REILLY \\ An PAN \\ Walter C. WILLETT \\ Ichiro KAWACHI \\ Karestan KOENEN \\ Alberto ASCHERIO \\ Harvard School of Public Health, \\ Harvard University, \\ 655 Huntington Avenue, \\ Boston, \\ MA 02115, \\ USA \\ $<$ mlucas@hsph.harvard.edu $>$
}

\begin{abstract}
A relative decrease intake of $n-3$ to $n-6$ fatty acids has been implicated in the pathogenesis of depression. However, the associations between different sources of dietary $n-3, n-6$, and their ratio, and the risk of depression have not been prospectively studied. We prospectively studied 54,632 women who were 50 to 77 years of age and free from depressive symptoms at baseline. Information on diet was obtained from validated food frequency questionnaires (FFQ) completed four times before baseline $(1984,1986,1990,1994)$. Clinical depression was defined as reporting both physiciandiagnosed depression and regular antidepressant medication use. Relative risks (RR) of clinical depression, adjusted for age and other possible risk factors, were estimate using Cox-proportional hazard models. During 10 years of follow-up (1996-2006), 2,823 incident cases of clinical depression were documented. Long-chain $n-3$ intake from fish was not associated with depression risk (multivariate RR for $0.3 \mathrm{~g} /$ day increment $=0.99$ [95\% Cl: 0.88, 1.10]). Intake of alpha-linolenic acid (ALA) was not associated with depression risk, except in multivariate model adjusted for $n-6$ linoleic acid (LA). For each $0.5 \mathrm{~g} /$ day increment of ALA, multivariate RR was 0.82 (95\% Cl, 0.71 to 0.94). Interaction between $A L A$ and $L A$ was found to be significant for depression risk $(P=0.02)$. Within quintiles of $L A$ a a $0.5 \mathrm{~g} /$ day increment in $A L A$ was inversely associated with depression in the first, second and third $L A$ quintiles ( $R R=0.57$ ( $95 \% \mathrm{Cl}, 0.37$ to 0.87 ); 0.62 (95\% Cl, 0.41 to 0.93 ); 0.68 ( $95 \% \mathrm{Cl}, 0.47$ to 0.96 ) respectively) but not in the fourth and fifth quintiles. The results of this large longitudinal study do not support a protective effect of long-chain n-3 from fish or fish intake on depression risk. However, this study provides support for the hypothesis that higher ALA and lower LA intakes might reduce depression risk, but this relation warrants further investigation.
\end{abstract}

Key words: depression, cohort, women, omega-3 fatty acids, alpha-linolenic acid, linoleic acid, EPA, DHA, fish

lorsqu'ils sont administrés comme adjuvant aux antidépresseurs (Freeman et al., 2006 ; Lin et Su, 2007 ; Appleton et al., 2010). Cependant, une hétérogénéité significative entre les études et un biais de publication ont été notés (Freeman et al., 2006, Lin et Su, 2007 ; Appleton et al., 2010). À ce jour, seulement 3 études de cohortes ont analysé cette relation (Hakkarainen et al., 2004; SanchezVillegas et al., 2007 ; Colangelo et al., 2009). Les résultats des précédentes études de cohortes prospectives semblent contradictoires en raison de limitations dans le design des études, plus particulièrement une évaluation des apports alimentaires uniquement au

\begin{tabular}{|c|c|}
\hline & Abréviations \\
\hline $\mathrm{AA}$ & acide arachidonique \\
\hline AGPI & acide gras polyinsaturé \\
\hline AGPI-LC & $\begin{array}{l}\text { acides gras polyinsaturés à } \\
\text { longues chaînes }\end{array}$ \\
\hline ALA & acide alphalinolénique \\
\hline $\mathrm{DHA}$ & acide docosahexaénoïque \\
\hline DPA & acide docosapentaénoïque \\
\hline EPA & acide eicosapentaénoïque \\
\hline FFQ & $\begin{array}{l}\text { questionnaire de } \\
\text { fréquence alimentaire }\end{array}$ \\
\hline IC à $95 \%$ & $\begin{array}{l}\text { intervalles de confiance } \\
\text { à } 95 \%\end{array}$ \\
\hline IMC & indice de masse corporelle \\
\hline
\end{tabular}

Pour citer cet article : Lucas M, Mirzaei F, O'Reilly ÉJ, Pan A, Willett WC, Kawachi I, Koenen K, Ascherio A. Relation entre n-3 et n-6 avec la dépression clinique : résultats de la Nurses' Health Study. OCL $2011 ; 18(4)$ : 181-7. doi : 10.1684/ocl.2011.0394 


\begin{tabular}{|ll|}
\hline LA & acide linoléique \\
MHI-5 & Mental Health Index \\
$\mathrm{NHS}$ & Nurses' Health Study \\
$\mathrm{n}-3$ & oméga-3 \\
$\mathrm{n}-6$ & oméga-6 \\
$\mathrm{RR}$ & risques relatifs \\
$\mathrm{SDS}$ & symptômes dépressifs \\
& sévères \\
\hline
\end{tabular}

début de la période de suivi, une taille d'échantillon réduite, les mesures utilisées pour définir la dépression et une courte durée de suivi.

Les principales sources d'AGPI dans I'alimentation et les tissus humains sont I'acide linoléique (LA, 18:2 n-6) et l'acide alphalinolénique (ALA, 18:3 n-3), qui représentent respectivement $\sim 89 \%$ $(\sim 15 \mathrm{~g} / \mathrm{j})$ et $\sim 9 \%(\sim 1,5 \mathrm{~g} / \mathrm{d})$ des apports en AGPI aux États-Unis (KrisEtherton et al., 2000 ; What We Eat in America, 2005-2006). Les AGPI LA et ALA exigent les mêmes enzymes métaboliques pour former leurs dérivés à longues chaînes n- 6 et $n-3$ (acide arachidonique (AA, 20:4 n-6), docosapentaénoïque (DPA, 22:5 n-6), acide eicosapentaénoïque (EPA, 20:5 n-3), acide docosapentaénoïque (DPA, 22:5 $\mathrm{n}-3)$, acide docosahexaénoïque (DHA, 22:6 n-3)). II a été observé que l'apport en LA pourrait réduire l'incorporation de I'ALA dans les tissus et/ou sa conversion en n-3 à longues chaînes (Goyens et al., 2006 ; Liou et al., 2007, Ghosh et al., 2007).

Bien que les $n-3$ soient souvent associés à un risque moindre de symptômes dépressifs, peu d'études ont analysé les associations entre les différents types $d^{\prime}$ acides gras $n-3, n-6$, et leur ratio en relation avec la dépression clinique. Seulement une étude a examiné la relation entre I'ALA et la dépression (Hakkarainen et al., 2004). Nous avons donc examiné auprès des participantes de la Nurses' Health Study (NHS), la relation entre les différents types $d^{\prime}$ acides gras $n-3$ et $n-6$ avec le risque d'incidence de dépression clinique.

\section{Méthodes}

\section{Population sous étude}

La NHS est une cohorte prospective de 121700 infirmières américaines âgées de 30 à 55 ans au moment du recrutement (1976). Tous les 2 ans, les participantes ont fourni, au moyen de questionnaires administrés par la poste, des informations sur le style de vie, les antécédents médicaux et les nouveaux diagnostics médicaux. Pour la présente analyse, la période de suivi a commencé au retour du questionnaire de 1996 et s'est terminée en juin 2006. Les femmes ont d'abord été invitées à déclarer leur utilisation d'antidépresseur en 1996 et leur histoire de diagnostic de dépression par un médecin en 2000. Afin d'analyser de manière prospective la relation entre les différents $n-3$ et $n-6$ et l'incidence de la dépression, les femmes qui ont déclaré en 1996 utiliser un antidépresseur ou avoir eu un épisode de dépression diagnostiqué par un médecin en 1996 (ou avant) ont été exclues de la population à l'étude. Les femmes qui ont présenté des symptômes dépressifs sévères (SDS) (score $\leq 52$ ) en 1992 ou 1996 au questionnaire Mental Health Index (MHI-5) (28-30), une sous-échelle de 5 points du Short-Form (SF-36) Health Status Survey, ont été également exclues. Les femmes qui n'ont pas déclaré leur statut de dépression en 1996, 1998 ou 2000, ou qui n'ont pas retourné ou répondu au MHI-5 en 1992 ou 1996 ont été exclues parce que leur histoire de dépression en 1996 n'a pu être établie. En 2000, 76516 femmes étaient en vie, et ont complété les périodes relatives aux questionnaires de 1996 à 2000. De celles-ci, 64246 ont été considérées comme sans dépression en 1996 et constitue donc la population sous étude pour la présente analyse. Après I'exclusion de celles qui avaient des valeurs manquantes pour les variables d'exposition ( $\mathrm{N}=9614$ ), la population finale de 1996 comprend 54632 femmes. Le protocole de l'étude a été approuvé par I'Institutional Review Boards of Brigham and Women's Hospital and the Harvard School of Public Health.

\section{Évaluation nutritionnelle}

En 1980, un questionnaire de fréquence alimentaire (FFQ) comprenant 61 questions, contenant une seule question sur la consommation de poisson, a été administré pour évaluer la consommation de matières grasses et $d$ 'autres nutriments spécifiques (Willett et al., 1985). En 1984, ce FFQ a été révisé pour intégrer 116 questions sur différents aliments dont 4 questions portant sur les produits marins (poisson à viande brune, thon en conserve, autres poissons, mollusques et crustacés). La reproductibilité et la validité des FFQ (Willett et al., 1988), ainsi que le calcul de I'apport en acides gras EPA+DHA, ont été publiés ailleurs (Iso et al., 2001). Puisque les FFQ ultérieurs contenaient des aliments critiques à l'évaluation des apports en $n-3$ et n-6, nous avons donc considéré le FFQ de 1984 comme point de départ pour toutes les informations relatives aux nutriments. Ces questionnaires élargis incorporent aussi d'autres questions concernant la marque de margarine et les types de matières grasses utilisées pour la cuisson. Des questions ont également été posées concernant la fréquence d'utilisation de mayonnaise ou d'autres vinaigrettes crémeuses et de vinaigrette à base d'huile pour la salade. La composition nutritionnelle en acides gras de la vinaigrette et la mayonnaise a été imputée par celle de I'huile de soja, qui représentait tous (vinaigrette) ou la plupart (mayonnaise) des produits disponibles dans les marchés jusqu'à tout récemment. La composition en ALA et les autres nutriments ont été obtenues à partir de la Harvard University Food Composition Database provenant du US Department of Agriculture sources (US Department of Agriculture, 1989) et complétée par I'analyse directe d'échantillons d'aliments obtenus à partir de magasins, d'épiceries et de restaurants fast-food de la région Boston. Plus de détails ont été publiés ailleurs (Willett et Stampfer, 1986).

Tous les apports en nutriments ont été ajustés pour l'apport énergétique total, selon le modèle résiduel (Willett et Stampfer, 1986). Comme mesure substitutive de l'exposition alimentaire à long terme, nous avons pris la moyenne cumulative de 4 évaluations alimentaires $(1984,1986,1990,1994)$ précédant notre point de départ du suivi (1996). Cette moyenne représente mieux les apports alimentaires à long terme qu'une seule évaluation des apports au début de la période de suivi et est beaucoup moins susceptible d'être affectée par la causalité inverse que celle mesurée par les apports alimentaires plus récents (updated dietary intake).

\section{Détermination des cas}

La dépression clinique a été définie comme avoir rapporté un nouveau diagnostic de dépression par un médecin et une prise régulière $d^{\prime}$ 'antidépresseur. 
En 2000, les participants ont été invités à indiquer l'année de leur premier diagnostic de dépression par un médecin ( $\leq 1996,1997,1998,1999,2000)$. Par la suite, cette information a été mise à jour tous les deux ans jusqu'en 2006. La question sur l'utilisation régulière d'antidépresseur a été posée en 1996, et cette information a été mise à jour à tous les deux ans jusqu'en 2006. Ainsi, le cycle du questionnaire de 1996 a été considéré comme point de référence pour le début de la période de suivi.

\section{Analyse statistique}

Parmi les femmes qui étaient sans SDS au début de la période de suivi, les personne-années de suivi ont été calculées à partir de la date de retour du questionnaire de 1996 jusqu'au premier événement à survenir, soit la mort, le $1^{\mathrm{er}}$ juin 2006, ou la date de retour de leur dernier questionnaire. Le modèle de risques proportionnels de Cox, stratifié pour l'âge en mois et le cycle du questionnaire, a été utilisé pour estimer les risques relatifs (RR) et leurs intervalles de confiance à $95 \%$ (IC à $95 \%)$ de développer une dépression clinique. Le choix des unités pour exprimer les RR des apports en n-3 et n-6 (traités en continu) a été basé sur les différences entre les $90^{\mathrm{e}}$ et $10^{\mathrm{e}}$ percentiles de leurs apports moyens cumulatifs. Les analyses multivariées ont d'abord été ajustées pour les covariables non alimentaires, soit le statut hormonal (post-ménopause avec ou sans traitement hormonal, préménopause), l'origine ethnique (caucasienne ou non), l'obésité (indice de masse corporelle (IMC) $\geq 30 \mathrm{~kg} / \mathrm{m}^{2}$, oui/non), statut tabagique (jamais fumé, fumeur passé, fume actuellement 1-14 cig./j, 15-24 cig./j, ou $\geq 25$ cig./j), l'activité physique (quintiles), un diagnostic de diabète, de cancer, d'infarctus du myocarde ou $d^{\prime}$ angine de poitrine, et l'utilisation de multivitamines (oui/non) (modèle 1). Toutes les covariables non alimentaires ont été mises à jour à tous les deux ans. Nous avons ensuite ajusté les modèles multivariés pour les moyennes cumulatives des covariables alimentaires (tous traités comme variables en continues), soit l'apport énergétique $(\mathrm{kcal} / \mathrm{j})$, l'alcool $(\mathrm{g} / \mathrm{j})$, les protéines $(\mathrm{g} / \mathrm{j})$, les acides gras trans $(\mathrm{g} / \mathrm{j})$, saturés $(\mathrm{g} / \mathrm{j})$, mono-insaturés $(\mathrm{g} / \mathrm{j})$, les autres $\mathrm{AGPI}$ $\mathrm{n}-3$ et $\mathrm{n}-6(\mathrm{~g} / \mathrm{j})$, et la consommation $\mathrm{d}^{\prime}$ huile de poisson (jamais, seulement en 1990, seulement en 1994, en 1990 et 1994) (modèle 2).

Nous avons également calculé la moyenne cumulative de 1984 à 1994 pour la consommation de total de poisson. La modélisation des risques proportionnels de Cox a également été adoptée pour estimer le RR (IC à $95 \%$ ) de développer une dépression clinique en fonction de la fréquence de consommation de poisson. Les participantes ont été classées en 5 groupes : $<1$ fois par mois (groupe de référence), 1-3 fois par mois, 1 fois par semaine, 2-4 fois par semaine, et $\geq 5$ fois par semaine.

L'interaction entre I'apport en ALA et LA a été évaluée en utilisant le produit croisé des 2 termes, avec les 2 éléments traités comme variables continues. L'interaction entre l'apport en ALA et LA a été jugée positivement significative en regard du risque de dépression clinique $(P=0,02)$. Par conséquent, I'association entre I'apport alimentaire d'ALA et le risque de dépression clinique a également été évaluée séparément dans chaque quintile de LA, et vice versa. Toutes les analyses ont été réalisées avec le logiciel SAS, version 9.1 (SAS Institute Inc, 2003). Toutes les valeurs $P$ sont bilatérales.

\section{Résultats}

Parmi les 54632 femmes qui étaient sans SDS au début de la période du suivi, 2823 nouveaux cas de dépression clinique ont été enregistrées durant 10 années de suivi (495 829 personnesannées). Comme il a été indiqué en détail ailleurs (Lucas et al., 2011), les femmes du quintile supérieur de consommation d'ALA étaient moins actives physiquement et avaient une plus forte prévalence de tabagisme, d'hypertension, de diabète et d'utilisation d'huile de poisson comparativement à celles du $1^{\mathrm{er}}$ quintile. En comparaison au $1^{\text {er }}$ quintile de l'apport en EPA+ $\mathrm{DHA}$, les femmes du quintile supérieur étaient légèrement plus âgées, plus actives et avaient une plus faible prévalence de tabagisme, mais une prévalence plus élevée de cancer, d'hypertension, $d^{\prime}$ utilisation d'huile de poisson et de multivitamines. Les apports médians journaliers, ajustés pour l'apport énergétique, variaient de $0,75 \mathrm{~g}$ pour le quintile le plus bas d'ALA à 1,20 g pour le quintile le plus élevé. Pour les apports en EPA+DHA, ceux-ci variaient de 0,08 à $0,37 \mathrm{~g}$ pour le $1^{\mathrm{er}}$ et dernier quintile, respectivement. Sur la base des apports déclarés au FFQ de 1984, les plus importants contributeurs (en \% absolu) à l'apport global d'ALA étaient la mayonnaise ou autres vinaigrettes crémeuses $(16,7 \%)$, la vinaigrette à base d'huile pour la salade (12,2\%), la margarine $(6,8 \%)$, la viande de bœuf, de porc et d'agneau comme plat principal (6,5 \%), les aliments frits consommés à la maison ou à l'extérieur de la maison (4,4\%), le lait $(4,1 \%)$ et le beurre $(3,4 \%)$. Pour I'apport en LA, les plus importants contributeurs étaient la mayonnaise ou autres vinaigrettes crémeuses (14,6\%), la margarine $(9,8 \%)$, la vinaigrette à base $d^{\prime}$ huile pour la salade $(9,1 \%)$, les aliments frits consommés à la maison ou à l'extérieur de la maison $(7,1 \%)$, les croustilles (5,5\%), les noix $(4,7 \%)$ et le beurre d'arachide (4,1\%).

Les apports en $n-3$ de sources végétales, ALA, n'étaient pas associés au risque de dépression dans le modèle ajusté pour I'âge ni celui ajusté pour les covariables non alimentaires (modèle 1) (figure 1). Cependant, lorsque nous avons ajusté pour les facteurs alimentaires (modèle 2 ), le RR de dépression clinique était de 0,82 (IC $95 \%$ : 0,71 à 0,94) pour chaque augmentation de 0,5 g/j d'ALA. L'apport alimentaire d'EPA+DHA provenant des produits marins (figure 1) ou la fréquence de consommation de poisson (figure 2) n'était pas associé au risque de dépression clinique. Lorsque nous avons examiné séparément les types de poissons, ni la consommation de poisson gras ni celle des poissons maigres a été associée significativement au risque de dépression.

Le risque de dépression clinique augmentait avec un apport accru en LA (pour chaque augmentation de $5 \mathrm{~g} / \mathrm{j}$, le $\mathrm{RR}=1,26 ; 95 \% \mathrm{Cl}: 1,07$ à 1,49$)$ (modèle 2, figure 1) et diminuait avec une augmentation du ratio ALA/LA ( $P$ de tendance $<0,001$ ) et le ratio n-3/n-6 ( $P$ de tendance $=0,004)$. La consommation d'acide arachidonique (AA) n'était pas été associée de façon significative ( $P$ $=0,80$ ) avec le risque de dépression dans le $2^{\mathrm{e}}$ modèle multivarié.

Nous avons aussi évalué le risque de dépression clinique pour les apports en ALA et LA dans les quintiles de LA et ALA, respectivement. Pour chaque augmentation de $0,5 \mathrm{~g} / \mathrm{j}$ d'ALA, le RR de dépression clinique était de 0,57 (IC $95 \%$ : 0,37 à 


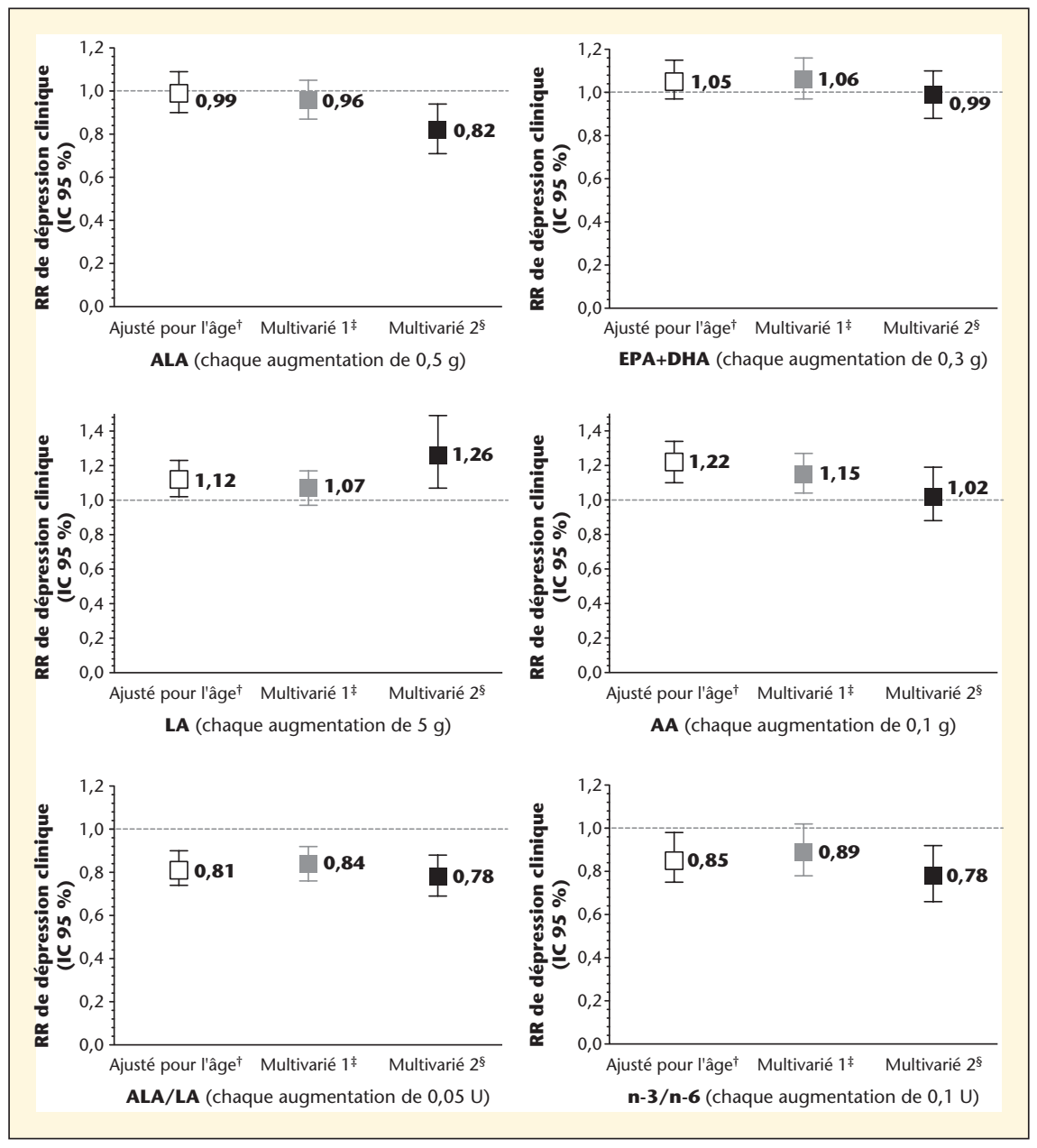

Figure 1. Risques relatifs $(R R)$ de dépression clinique en fonction des apports en acides gras $n-3$ et $n-6$ chez les femmes de la Nurses' Health Study*

*Les valeurs sont les RR (IC à $95 \%$ ) estimés à partir du modèle de risques proportionnels de Cox. Le RR est pour chaque augmentation de l'unité d'acides gras ou des ratios. AA, acide arachidonique; $A L A$, acide alphalinolénique; $D H A$, acide docosahexaénö̈que, EPA, acide eicosapentaénoïque, $L A$, acide linoléique.

${ }^{\dagger}$ Modèle ajusté pour l'âge (en mois) et l'intervalle de temps.

${ }^{\ddagger}$ Modèle ajusté en plus pour le statut hormonal (postménopause avec ou sans traitement hormonal, en préménopause), l'origine ethnique (caucasienne ou non), l'obésité (indice de masse corporelle (IMC) $\geq 30 \mathrm{~kg} / \mathrm{m}^{2}$, oui/non), statut tabagique (jamais fumé, fumeur passé, fume actuellement 1-14 cig./j, 15-24 cig./j, ou $\geq 25 \mathrm{cig} . / \mathrm{j})$, I'activité physique (en quintiles), un diagnostic de diabète (binaire), de cancer (binaire), l'infarctus du myocarde ou l'angine de poitrine (binaire), et l'utilisation de multivitamines (binaire).

¿Modèle ajusté en plus pour l'apport cumulatif moyen (1984-94) de l'énergie (kcal/j), des protéines $(g / j)$, des acides gras trans $(g / j)$, saturés $(g / j)$, mono-insaturés $(g / j)$, de l'alcool $(g / j)$ et I'utilisation d'huile de poisson (jamais, seulement en 1990, seulement en 1994, en 1990 et 1994). Pour l'ALA, le modèle 2 a été ajusté en plus pour I'EPA+DHA $(g / j), L A(g / j), A A(g / j)$. Pour I'EPA+DHA, le modèle 2 a également été ajusté pour I'ALA, $L A, A A$. Pour $L A$, le modèle 2 a également été ajusté pour I'ALA, EPA + DHA, $A A$. Pour $A A$, le modèle 2 a également été ajusté pour I'ALA, EPA + DHA, LA. Pour le ratio ALA/LA, le modèle 2 a été ajusté pour I'EPA $+D H A, A A$

$0,87)$ dans le $1^{\text {er }}$ quintile de $L A$, de 0,62 (IC $95 \%: 0,41$ à 0,93 ) dans le $2^{\mathrm{e}}$, de 0,68 (IC $95 \%: 0,47$ à 0,96) dans le $3{ }^{\mathrm{e}}$, de 0,90 (IC $95 \%: 0,66$ à 1,23) dans le $4^{\mathrm{e}}$, et de 1,09 (IC $95 \%: 0,91$ à 1,32 ) dans le $5^{\mathrm{e}}$ quintile (données non présentées). Pour chaque augmentation de $5 \mathrm{~g} / \mathrm{j}$ de LA, le RR de dépression clinique était de 1,04 (IC $95 \%: 0,73$ à 1,49$)$ dans le $1^{\mathrm{er}}$ quintile d'ALA, de 1,07 (IC $95 \%: 0,71$ à 1,62) dans le $2^{\mathrm{e}}$, de 1,26 (IC $95 \%: 0,85$ à 1,86) dansle $3^{\mathrm{e}}$, de 1,71 (IC $95 \%: 1,16$ à 2,52) dans le $4^{\mathrm{e}}$, et de 1,22 (IC $95 \%: 0,93$ à 1,59 ) dans le $5^{\mathrm{e}}$ quintile (données non présentées).

\section{Discussion}

Auprès de cette importante cohorte prospective de femmes, nous avons constaté qu'un apport alimentaire élevé en $n-3$ d'origine végétale, ALA, était significativement associé à un risque plus faible de dépression clinique, en particulier parmi celles qui avaient une faible consommation en LA. Nous n'avons pas observé d'association entre le risque de dépression clinique et la fréquence de consommation de poisson ou l'apport en EPA+DHA. Un nouvel aspect de notre étude est l'analyse plus complète des AGPI n-3 et n-6 et de leur impact sur le risque de dépression et, par conséquent, I'interaction significative observée entre LA et ALA. Alors que les précédentes études longitudinales ont étudié la relation entre les $n-3$ et la prévalence de dépression, la contribution unique de notre étude est qu'elle examine le risque $d^{\prime}$ 'incidence de dépression avec des avantages supplémentaires qui sont un suivi à long terme, I'utilisation de multiples évaluations de l'apport alimentaire et de la dépression, un échantillon de grande taille, l'ajustement pour des facteurs de risque mis à jour à tous les 2 ans, et une définition plus rigoureuse de la dépression clinique.

Puisque les acides gras ALA et LA exigent des enzymes métaboliques communs, jusqu'à $11,5 \%$ de I'ALA peut être converti en EPA lorsque l'apport en LA est faible (Goyens et al., 2006). Par conséquent, la capacité de I'ALA à augmenter légèrement les niveaux des AGPI-LC n-3, principalement I'EPA, et dans une moindre mesure le DPAn-3, pourrait expliquer en partie nos résultats. Certains auteurs ont suggéré que I'EPA seul ou un ratio supérieur EPA/DHA est associé à de meilleurs résultats qu'une supplémentation constituée seulement de DHA (Freeman et al., 2006 ; Martins, 2009). Un effet protecteur de l'EPA serait compatible avec nos résultats observés avec l'apport en ALA, dont très peu est converti en DHA (Plourde et Cunnane, 2007). Un argument contre l'effet de I'ALA par l'entremise de sa conversion en EPA est l'absence de relation notée 


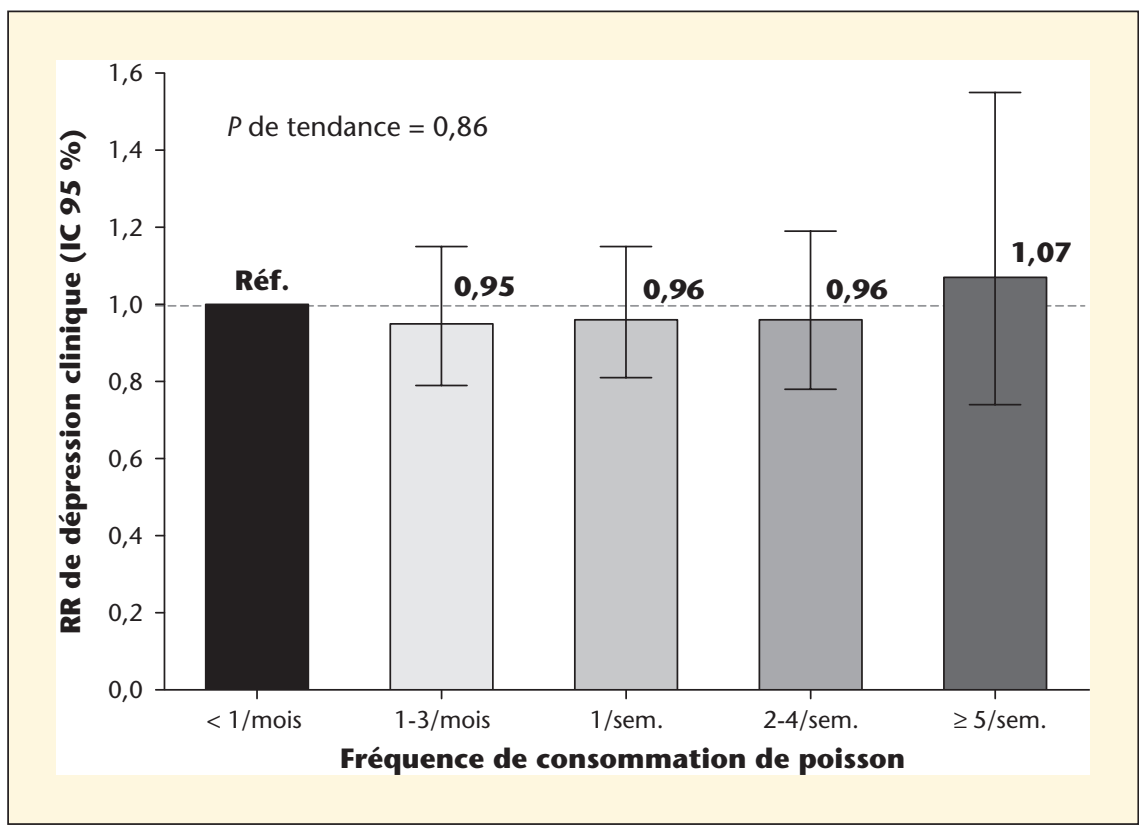

Figure 2. Risques relatifs $(R R)$ de dépression clinique en fonction de la fréquence de consommation de poisson chez les femmes de la Nurses' Health Study*.

*Les valeurs sont les RR (IC à $95 \%$ ) estimés à partir du modèle de risques proportionnels de Cox. Modèle ajusté pour l'âge (en mois), l'intervalle de temps, le statut hormonal (postménopause avec ou sans traitement hormonal, en préménopause), l'origine ethnique (caucasienne ou non), l'obésité (indice de masse corporelle (IMC) $\geq 30 \mathrm{~kg} / \mathrm{m}^{2}$, oui/non), statut tabagique (jamais fumé, fumeur passé, fume actuellement 1-14 cig./j, 15-24 cig./j, ou $\geq 25$ cig./j), l'activité physique (en quintiles), un diagnostic de diabète (binaire), de cancer (binaire), l'infarctus du myocarde ou l'angine de poitrine (binaire), I'utilisation de multivitamines (binaire), I'apport cumulatif moyen (1984-94) de l'énergie $(\mathrm{kcal} / \mathrm{j})$, des protéines $(\mathrm{g} / \mathrm{j})$, des acides gras trans $(\mathrm{g} / \mathrm{j})$, saturés $(\mathrm{g} / \mathrm{j})$, monoinsaturés $(g / j)$ et de l'alcool $(g / j)$, de l'ALA $(g / j), L A(g / j)$, et $A A(g / j)$, et l'utilisation d'huile de poisson (jamais, seulement en 1990, seulement en 1994, en 1990 et 1994).

entre la consommation d'EPA+DHA et la dépression. Toutefois, I'apport en EPA des participantes est faible (médiane $=$ $58 \mathrm{mg} / \mathrm{j}$ ) et donc la formation d'EPA à partir de I'ALA alimentaire pourrait dépasser celle de l'apport en EPA. II est également possible qu'à long terme l'apport alimentaire en ALA puisse jouer un rôle physiologique dans la dépression clinique, indépendamment de I'EPA + DHA.

En dépit d'ajustement pour les covariables, y compris I'ALA, une augmentation de I'apport en LA était associée à un risque dépression clinique accru de $26 \%$ (95\% Cl : $7 \%$ à $49 \%$ ) (figure 1). Encore une fois, cela peut être dû à l'interaction significative entre I'ALA et LA. L'analyse de stratification auprès des quintiles d'ALA a indiqué que l'augmentation des apports en LA était seulement liée à un risque significativement plus élevé de dépression clinique chez les femmes dans le $4^{\mathrm{e}}$ quintile $\mathrm{d}^{\prime} \mathrm{ALA}$. Ce risque significativement accru est probable- été observée. Parmi les 7903 participants de la cohorte espagnole SUN, une réduction du risque de troubles mentaux a été notée dans le $2^{\mathrm{e}}$ et $4^{\mathrm{e}}$ quintile des apports en AGPI-LC n-3 en comparaison au $1^{\text {er }}$ quintile, après un suivi médian de 27,5 mois (SanchezVillegas et al., 2007). Toutefois, aucune tendance linéaire n'a été observée, et la plupart des cas (environ $67 \%$ ) étaient des troubles anxieux. Dans une cohorte américaine de 3317 hommes et femmes, les rapports de cote de symptômes dépressifs élevés après 3 ans de suivi n'étaient pas significativement différents entre les quintiles supérieurs et les $1^{\text {er }}$ quintiles d'apports en AGPI-LC $n-3$ ou de poisson, mesurés au début de la période de suivi (Colangelo et al., 2009). Cependant, une association inverse entre les apports en AGPI-LC n-3 et le nombre de visites pour des symptômes dépressifs a été notée seulement chez les femmes (Colangelo et al., 2009).

L'utilisation de 4 évaluations alimentaires sur une période de 10 ans est une force unique de notre étude. En effet, les autres cohortes ont complété seulement un FFQ au début de la période de suivi comme mesure d'exposition. Cette approche est moins précise que la nôtre car elle suppose que l'apport alimentaire mesuré une fois au début de la période de suivi représente adéquatement les habitudes alimentaires usuelles et qu'elles demeurent inchangées pour l'ensemble de la période de suivi. De plus, les apports en ALA et LA mesurés par nos FFQ ont été validés avec les mesures de ces acides gras dans les tissus adipeux $(r=0,34(P<0,001)$ pour l'ALA et $r=0,37(P<0,001)$ pour LA) (Garland et al., 1998). Toutefois, en raison du fait que les acides gras ALA et LA proviennent largement des produits alimentaires identiques ou similaires, la dissociation complète de l'apport en ALA et LA n'est pas possible, et un certain degré de classification erronée et de partialité en raison de confusion résiduelle est inévitable (Rosner et al., 1990). De plus, en raison d'une forte corrélation positive entre les apports en ALA et LA dans le régime alimentaire américain, le ratio LA/ALA affiche une variabilité modeste. II serait donc intéressant d'examiner la relation entre les apports spécifiques en acides gras et la dépression dans des populations où les habitudes alimentaires permettent une 
meilleure discrimination entre les acides gras ALA et LA. Les limitations statistiques communes aux études avec des comparaisons multiples s'appliquent également à la présente étude.

Une préoccupation importante dans les études épidémiologiques est la causalité inverse (reverse causation). Celle-ci peut résulter d'un effet des symptômes dépressifs sur le régime alimentaire. Par exemple, une fausse association inverse entre les apports en n-3 et le risque de dépression pourrait être observée si les femmes avec humeur dépressive ont réduit leur apport en $\mathrm{n}-3$. Pour minimiser le biais de cette source, nous avons exclu, au départ, 12270 femmes présentant des SDS, et nous avons considéré la moyenne cumulative des apports en n-3 et n- 6 entre 1984 et 1994 pour prédire la survenue de dépression entre 1996 et 2006 . Certaines erreurs de classification de la maladie est inévitable en raison d'une combinaison d'erreurs dans les autodéclarations de diagnostic de dépression ou la prise d'antidépresseur, le sous-diagnostic de la dépression par les médecins (Lowe et al., 2004), le sous-traitement de la dépression (Demyttenaere et al., 2004), et la prescription d'antidépresseur pour d'autres indications que la dépression. Nous avons essayé de maximiser la spécificité de la définition de nos cas, et par conséquent, accepter seulement comme nouveaux cas de dépression les femmes qui ont déclaré à la fois un diagnostic de dépression et I'utilisation d'antidépresseur. Un pourcentage important des cas de dépression diagnostiqués n'ont pas reçu d'antidépresseur durant le suivi. Ainsi, notre définition stricte de la dépression (i.e. diagnostic de dépression plus I'utilisation d'antidépresseur) a probablement causé une inclusion de cas relativement plus sévères. Dans la mesure où la probabilité de classer correctement les femmes avec une incidence de dépression est indépendante de leurs habitudes alimentaires (erreur de classification non différentielle de l'issue), la faible sensibilité de notre définition stricte des cas de dépression clinique ne devrait pas influencer les RR estimés (Rothman et Greenlet, 1998). Après 10 années de suivi, 5,2\% des femmes de notre cohorte ont développé une dépression clinique. Cette incidence n'est pas directement comparable à celle observée dans les populations non sélectionnées car pour réduire au minimum la causalité inverse, nous avons exclu les femmes présentant des SDS au début de la période de suivi, éliminant ainsi un groupe de femmes à haut risque de dépression. Ainsi, contrairement à la plupart des études précédentes, nos analyses de la NHS abordent le lien entre les apports en n-3 et n-6 et l'apparition de nouveaux cas de dépression relativement sévères, du moins assez graves pour être diagnostiqués comme tels et traités avec des antidépresseurs.

Plusieurs mécanismes biologiques pourraient potentiellement expliquer l'impact de I'ALA dans la dépression. Un déficit alimentaire en ALA a été lié à une biochimie altérée du cerveau, comme la structure et la fluidité de la membrane, les canaux ioniques, les seconds messagers, une réduction de la réponse du CREB (cAMP response element-binding protein transcription factor activity) et du BDNF (brain derived neurotrophic factor), une expression accrue de la phospholipase A2 cytosolique et sécrétoire et de la cyclo-oxygénase-2 (Yehuda et al., 1999 ; Haag, 2003 ; Rapoport et al., 2007). Les études animales ont également indiqué qu'une carence en ALA change la neurotransmission sérotoninergique et dopaminergique dans le cortex frontal (Delion et al., 1994 ; de la Presa Owens et Innis, 1999), et qu'une augmentation de sa consommation pourrait influencer la neurogenèse ainsi que les protéines clés impliquées dans les fonctions synaptiques (Blondeau et al., 2009). Les processus inflammatoires et la dysfonction endothéliale sont souvent impliqués dans la dépression et les maladies cardiovasculaires (Belmaker et Agam, 2008). Un apport alimentaire plus élevé en ALA a été associé à des niveaux plasmatiques bas de biomarqueurs inflammatoires et d'activation endothéliale chez les femmes de la NHS (Lopez-Garcia et al., 2004). Toutefois, les mécanismes d'actions des apports alimentaires en $n-3$ et $n-6$ dans la dépression méritent $d$ 'être approfondis chez les humains.

\section{Conclusion}

Les résultats de cette vaste étude longitudinale ne supportent pas un effet protecteur des AGPI-LC n-3 ou la consommation de poisson sur le risque de dépression. Des apports plus élevés en ALA et moins élevés en LA ont été associés à un risque plus faible de dépression clinique, mais cette association doit être interprétée avec prudence en raison de la difficulté de séparer les effets des apports en nutriments corrélés.

Remerciements. Nous sommes redevables aux participants de la Nurses' Health Study pour leur appui exceptionnel et continuel ainsi qu'à nos collègues de travail de l'étude pour leur précieuse aide. Soutenu par une subvention du National Institutes of Health (NIH) (DK58845). Le Dr Ascherio a reçu une subvention du National Alliance for Research on Schizophrenia \& Depression (Project ID : 5048070-01). Le Dr Lucas a reçu une bourse postdoctorale du Fonds de recherche en santé du Québec (FRSQ). Les sources de financement $\mathrm{n}^{\prime}$ ont pas été impliquées dans la collecte de données, l'analyse des données, la rédaction du manuscrit et la publication.

\section{REFERENCES}

Appleton KM, Rogers PJ, Ness AR. Updated systematic review and meta-analysis of the effects of $\mathrm{n}-3$ long-chain polyunsaturated fatty acids on depressed mood. Am / Clin Nutr $2010 ; 91$ : 757-70.

Belmaker RH, Agam G. Major depressive disorder. N Engl J Med 2008 ; 358 : 55-68.

Blondeau N, Nguemeni C, Debruyne DN, et al. Subchronic alpha-linolenic acid treatment enhances brain plasticity and exerts an antidepressant effect: a versatile potential therapy for stroke. Neuropsychopharmacology 2009 ; 34 : 2548-59.

Colangelo LA, He K, Whooley MA, Daviglus ML, Liu K. Higher dietary intake of long-chain omega-3 polyunsaturated fatty acids is inversely associated with depressive symptoms in women. Nutrition 2009; 25: 1011-9.

de la Presa Owens S, Innis SM. Docosahexaenoic and arachidonic acid prevent a decrease in dopaminergic and serotoninergic neurotransmitters in frontal cortex caused by a linoleic and alpha-linolenic acid deficient diet in formula-fed piglets. J Nutr 1999 ; 129 : 2088-93.

Demyttenaere K, Bruffaerts R, Posada-Villa J, et al. Prevalence, severity, and unmet need for treatment of mental disorders in the World Health Organization World Mental Health Surveys. JAMA 2004; 291 : 2581-90.

Delion S, Chalon S, Hérault J, Guilloteau D, Besnard JC, Durand G. Chronic dietary alpha- 
linolenic acid deficiency alters dopaminergic and serotoninergic neurotransmission in rats. J Nutr $1994 ; 124: 2466-76$.

Freeman MP, Hibbeln JR, Wisner KL, et al. Omega-3 fatty acids: evidence basis for treatment and future research in psychiatry. J Clin Psychiatry 2006 ; 67 : 1954-67.

Garland M, Sacks FM, Colditz GA, et al. The relation between dietary intake and adipose tissue composition of selected fatty acids in US women. Am J Clin Nutr 1998 ; 67 : 25-30.

Ghosh S, Novak EM, Innis SM. Cardiac proinflammatory pathways are altered with different dietary n-6 linoleic to n-3 alphalinolenic acid ratios in normal, fat-fed pigs. Am J Physiol Heart Circ Physiol 2007 ; 293 : H2919-27.

Goyens PL, Spilker ME, Zock PL, Katan MB, Mensink RP. Conversion of alpha-linolenic acid in humans is influenced by the absolute amounts of alpha-linolenic acid and linoleic acid in the diet and not by their ratio. $A m$ J Clin Nutr 2006 ; 84 : 44-53.

Haag M. Essential fatty acids and the brain. Can J Psychiatry 2003 ; 48 : 195-203.

Hakkarainen R, Partonen T, Haukka J, Virtamo J, Albanes D, Lonnqvist J. Is low dietary intake of omega-3 fatty acids associated with depression? Am J Psychiatry 2004; 161 : 567-9.

Hibbeln JR, Salem NJ. Dietary polyunsaturated fatty acids and depression: when cholesterol does not satisfy. Am J Clin Nutr 1995 ; 62:1-9.

Iso H, Rexrode KM, Stampfer MJ, et al. Intake of fish and omega-3 fatty acids and risk of stroke in women. JAMA 2001; 285 : 304-12.

Kessler RC, Berglund P, Demler O, et al. The epidemiology of major depressive disorder: results from the National Comorbidity Survey Replication (NCS-R). JAMA 2003; 289: 3095-105.

Kessler RC. Epidemiology of women and depression. J Affect Disord 2003 ; 74 : 5-13.

Kris-Etherton P, Shaffer Taylor D, Yu-Poth S, et al. Polyunsaturated fatty acids in the food chain in the United States. Am J Clin Nutr $2000 ; 71$ (suppl) : 179s-88s.

Lin PY, Su KP. A meta-analytic review of double-blind, placebo-controlled trials of antidepressant efficacy of omega- 3 fatty acids. J Clin Psychiatry 2007 ; 68 : 105661.

Liou YA, King DJ, Zibrik D, Innis SM. Decreasing linoleic acid with constant alpha-linolenic acid in dietary fats increases $(n-3)$ eicosapentaenoic acid in plasma phospholipids in healthy men. J Nutr 2007 ; 137 : 945-52.

Lopez-Garcia E, Schulze MB, Manson JE, et al. Consumption of (n-3) Fatty acids is related to plasma biomarkers of inflammation and endothelial activation in women. / Nutr 2004 ; 134 : 1806-11.

Lowe B, Spitzer RL, Grafe K, et al. Comparative validity of three screening questionnaires for DSM-IV depressive disorders and physicians' diagnoses. I Affect Disord 2004 ; $78: 131-40$.

Lucas M, Mirzaei F, O'Reilly EJ, et al. Dietary intake of $n-3$ and n- 6 fatty acids and the risk of clinical depression in women: a 10-y prospective follow-up study. Am / Clin Nutr 2011 ; 93 : 1337-43.

Martins JG. EPA but not DHA appears to be responsible for the efficacy of omega-3 long chain polyunsaturated fatty acid supplementation in depression: evidence from a meta-analysis of randomized controlled trials. J Am Coll Nutr 2009; 28 : 525-42.

Plourde M, Cunnane S. Extremely limited synthesis of long chain polyunsaturates in adults : implications for their dietary essentiality and use as supplements. Appl Physiol Nutr Metab 2007 ; 32 : 619-34.

Rapoport SI, Rao JS, Igarashi M. Brain metabolism of nutritionally essential polyunsaturated fatty acids depends on both the diet and the liver. Prostaglandins Leukot Essent Fatty Acids 2007 ; 77 : 251-61.

Rosner B, Spiegelman D, Willett WC. Correction of logistic regression relative risk estimates and confidence intervals for measurement error: the case of multiple covariates measured with error. Am / Epidemiol 1990 ; 132 : 734-45.

Rothman KJ, Greenland S. Modern Epidemiology. Philadelphia (PA) : Lippincott Williams \& Wilkins, 1998.

Simopoulos AP. Evolutionary aspects of diet, the omega-6/omega-3 ratio and genetic variation : nutritional implications for chronic diseases. Biomed Pharmacother 2006; 60 : 502-7.

Sanchez-Villegas A, Henriquez P, Figueiras A, Ortuno F, Lahortiga F, Martinez-Gonzalez MA. Long chain omega-3 fatty acids intake, fish consumption and mental disorders in the SUN cohort study. Eur J Nutr 2007 ; 46 : 337-46.

US Department of Agriculture. Composition of foods: raw, processed, and prepared, 1963. 1988. Agriculture handbook no.8. Washington, DC : US Government Printing Office. 1989.

What We Eat in America. National Health and Nutrition Examination Survey (NHANES) 2005-2006 [database on the Internet]. Beltsville, MD : USDA, Agricultural Research Service, Beltsville Human Nutrition Research Center, Food Surveys Research Group (Beltsville, MD and Hyattsville, MD) : U.S. Department of Health and Human Services, CDC, National Center for Health Statistics. Available from: http://www.ars.usda.gov/Services/docs.htm?docid=15044.

Willett WC, Sampson L, Stampfer MJ, et al. Reproducibility and validity of semiquantitative food frequency questionnaire. $A m$ J Epidemiol 1985; 122 : 51-65.

Willett W, Stampfer MJ. Total energy intake: implications for epidemiologic analyses. Am J Epidemiol 1986 ; 124 :17-27.

Willett WC, Sampson L, Browne ML, et al. The use of a self-administered questionnaire to assess diet four years in the past. Am J Epidemiol $1988 ; 127: 188-99$.

Yehuda S, Rabinovitz S, Mostofsky DI. Essential fatty acids are mediators of brain biochemistry and cognitive functions. J Neurosci Res 1999 ; 56 : 565-70. 